Jânio Monteiro · António João Silva ·

António Mortal · Jaime Aníbal ·

Manuela Moreira da Silva · Miguel Oliveira .

Nelson Sousa Editors
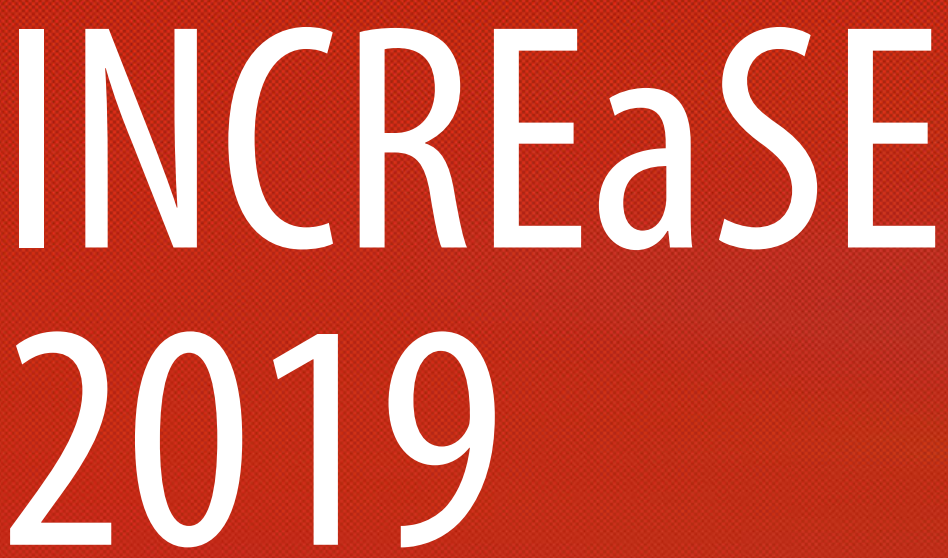

Proceedings of the 2nd International Congress on Engineering and Sustainability in the XXI Century 
INCREaSE 2019 
Jânio Monteiro · António João Silva ·

António Mortal · Jaime Aníbal .

Manuela Moreira da Silva .

Miguel Oliveira $\cdot$ Nelson Sousa

Editors

\section{INCREaSE 2019}

Proceedings of the 2nd International Congress on Engineering and Sustainability in the XXI Century 
Editors

Jânio Monteiro

Instituto Superior de Engenharia

Universidade do Algarve

Faro, Portugal

\section{António Mortal}

Instituto Superior de Engenharia

Universidade do Algarve

Faro, Portugal

Manuela Moreira da Silva

Instituto Superior de Engenharia

Universidade do Algarve

Faro, Portugal

Nelson Sousa

Instituto Superior de Engenharia

Universidade do Algarve

Faro, Portugal
António João Silva

Instituto Superior de Engenharia

Universidade do Algarve

Faro, Portugal

Jaime Aníbal

Instituto Superior de Engenharia

Universidade do Algarve

Faro, Portugal

Miguel Oliveira

Instituto Superior de Engenharia

Universidade do Algarve

Faro, Portugal

\author{
ISBN 978-3-030-30937-4 ISBN 978-3-030-30938-1 (eBook) \\ https://doi.org/10.1007/978-3-030-30938-1
}

(C) Springer Nature Switzerland AG 2020

This work is subject to copyright. All rights are reserved by the Publisher, whether the whole or part of the material is concerned, specifically the rights of translation, reprinting, reuse of illustrations, recitation, broadcasting, reproduction on microfilms or in any other physical way, and transmission or information storage and retrieval, electronic adaptation, computer software, or by similar or dissimilar methodology now known or hereafter developed.

The use of general descriptive names, registered names, trademarks, service marks, etc. in this publication does not imply, even in the absence of a specific statement, that such names are exempt from the relevant protective laws and regulations and therefore free for general use.

The publisher, the authors and the editors are safe to assume that the advice and information in this book are believed to be true and accurate at the date of publication. Neither the publisher nor the authors or the editors give a warranty, expressed or implied, with respect to the material contained herein or for any errors or omissions that may have been made. The publisher remains neutral with regard to jurisdictional claims in published maps and institutional affiliations.

This Springer imprint is published by the registered company Springer Nature Switzerland AG The registered company address is: Gewerbestrasse 11, 6330 Cham, Switzerland 


\section{Preface}

It is our pleasure to present the proceedings of the 2nd International Congress on Engineering and Sustainability in the XXI Century, INCREaSE 2019. INCREaSE is a multiple-track congress consisting of high-quality, previously unpublished papers, intended to act as a forum for research groups, engineers, and practitioners to present recent results, improvements, and promising future directions in engineering and sustainable development.

The contribution to the engineering and sustainable development is made in a multidisciplinary way, reflected by research and technology in the fields of Civil, Electronics, Food, and Mechanical Engineering. The work presented in the event includes several transversal challenges, such as Sustainable Development and Circular Economy, Climate Changes and Environmental Protection, Sustainable Technologies, Ocean and Coastal Technologies, Renewable Energy and Energy Efficiency, Water for Ecosystems and Societies, Healthy Food: Production, Characterization and Innovation, Sustainable Tourism, Natural and Anthropogenic Risks, Smart Cities, Smart Mobility and Accessibility, and Nearly Zero-Energy Buildings.

This year's INCREaSE was held during October 9-11, 2019, in Faro, Portugal, organized by the Institute of Engineering and hosted by University of Algarve. There was a very positive response to the Call for Papers for INCREaSE 2019. We received 172 works, from 481 different authors, from 21 countries, being accepted for publication 93 papers, where at least two reviewers reviewed each paper.

The essential actors of any conference are the authors, who submitted their scientific contributions. They are the main responsible for the high quality of the scientific program of INCREaSE 2019. The reviewers, members of the Scientific Committee and all the other committees, also played a key role with their dedicated and thorough work.

INCREaSE 2019 had an excellent group of invited speakers: Andrew Patton McCoy (Virginia Polytechnic Institute and State University, USA), Edmilson Teixeira (Federal University of Espírito Santo State, Brazil), Filipe Duarte do Santos (Faculty of Sciences, University of Lisbon, Portugal), and Tiago Rebelo (CEIIA, Portugal). We are grateful to these leading experts for their inspiring participation in INCREaSE 2019. 
We wish to express our gratitude to all of the above participants that enabled the success of the second edition of INCREaSE.

Finally, we look forward to meeting you again in the next edition of INCREaSE, in 2021.

October 2019

Jânio Monteiro

António João Silva

António Mortal

Jaime Aníbal

Manuela Moreira da Silva

Miguel Oliveira

Nelson Sousa 


\section{Organization}

INCREaSE is an international congress organized by the Engineering Institute from the University of Algarve, Faro, Portugal.

\section{Coordinating Committee}

António Mortal (Director)

Manuela Moreira da Silva

(President of the

Technical-Scientific Council)

João Ataíde

(Portuguese Secretary of State

for the Environment)

Paulo Águas (Rector)

Adelino Canário

Anabela Romano

António Ruano

António Tadeu

Armando Silva Afonso

Augusto Ferreira Guedes

Carlos Alberto Aires

Francisco Serra

João Guerreiro

Joaquim Peres

Luciano Lourenço

Luís Chícharo

Maria João Bebianno

Patrícia Pinto

Rogério Bacalhau
Universidade do Algarve, Instituto Superior de Engenharia, Portugal

Universidade do Algarve, Instituto Superior de Engenharia, Portugal

Ministry of the Environment and Energy

Transition, Portugal

Universidade do Algarve, Portugal

CCMAR, Portugal

MeditBio, Portugal

CINTAL, Portugal

ITeCons, Portugal

ANQIP, Portugal

Ordem dos Engenheiros Técnicos, Portugal

Ordem dos Engenheiros, Portugal

CCDR Algarve, Portugal

CRIA, Portugal

Águas do Algarve, S.A., Portugal

RISCOS, Portugal

UNESCO Chair in Ecohydrology, Portugal

CIMA, Portugal

CIEO, Portugal

C. M. Faro, Portugal 
Susana Neto

Vítor Aleixo

\section{Organizing Committee}

António João Silva

Elisa Silva

Isabel Ratão

Jaime Aníbal

Jânio Monteiro

Miguel Oliveira

Nelson Sousa

Paulo Santos

André Botelheiro (Director of Communication Office)

Ana Paula Ferreira (Director of Technical Services)

\section{Invited Speakers}

Andrew Patton McCoy

Edmilson Teixeira

Filipe Duarte do Santos

Tiago Rebelo

Isabel Ferreira

Stephen Thomas

\section{Scientific Committee}

Aicha Nancib

Aires Camões
APRH, Portugal

C. M. Loulé, Portugal

Universidade do Algarve, Instituto Superior de Engenharia, Portugal

Universidade do Algarve, Instituto Superior de Engenharia, Portugal

Universidade do Algarve, Instituto Superior de Engenharia, Portugal

Universidade do Algarve, Instituto Superior de Engenharia, Portugal

Universidade do Algarve, Instituto Superior de Engenharia, Portugal

Universidade do Algarve, Instituto Superior de Engenharia, Portugal

Universidade do Algarve, Instituto Superior de Engenharia, Portugal

Universidade do Algarve, Instituto Superior de Engenharia, Portugal

Universidade do Algarve, Portugal

Universidade do Algarve, Portugal

Virginia Polytechnic Institute and State University, USA

Federal University of Espírito Santo State, Brazil

Faculty of Sciences, University of Lisbon, Portugal

CEIIA, Portugal

Instituto Politécnico de Bragança, Portugal

OGI, Groundwater Specialists Ltd.,

Durham University, UK

Ferhat Abbas University, Setif 1, Algeria

University of Minho, Portugal 
Ana Cristina Figueira

Ana Teresa Lima

Anabela Rebelo

Andrew McCoy

Antonio Illana

António Martins

António Matias

António Ruano

Arturo Aquino Martín Aureliano Alves

Aurizia Anica

Carla Antunes

Carla Rodrigues

Carlos Otero da Silva

Celestina Pedras

Celestino Ruivo

Célia Quintas

Célia Ramos

Clauciana Schmidt Bueno de Moraes

Cláudia de Almeida

Cláudia Sequeira

Cristiano Cabrita

Damian Beben

Duarte Nuno Duarte

Edmilson Teixeira

Eduardo Esteves
Universidade do Algarve, ISE and CIEO, Portugal

Universidade Federal do Espírito Santo, Brasil and University of Waterloo, ON, Canada

Agência Portuguesa do Ambiente, Portugal

Virginia Polytechnic Institute and State University, USA

Universidad de Cádiz, Spain

Grupo Águas de Portugal, Águas do Algarve, Portugal

Universidade do Algarve, FE and CIEO, Portugal

Universidade do Algarve, FCT and CINTAL, Portugal

University of Huelva, Spain

Universidade do Algarve, FCT and CCMAR, Portugal

Universidade do Algarve, ESEC and IELT, Portugal

Universidade do Algarve, FCT and MeditBio, Portugal

Universidade de Aveiro e ANQIP, Portugal

Universidade do Algarve, ISE, Portugal

Universidade do Algarve, FCT and LEAF, Portugal

Universidade do Algarve, ISE, Portugal

Universidade do Algarve, ISE and MeditBio, Portugal

Universidade do Algarve, ESGHT, Portugal

Universidade Estadual Paulista, Brazil

Universidade do Algarve, ESGHT and CIEO, Portugal

Universidade do Algarve, ISE and CIMA, Portugal

Universidade do Algarve, ISE, Portugal

Opole University of Technology, Poland

Universidade do Algarve, FCT and CIMA, Portugal

Universidade Federal do Espírito Santo, Brazil

Universidade do Algarve, ISE and CCMAR, Portugal 
Eduardo López González

Eladio Durán Aranda

Enzo Martinelli

Eugénia Ferreira

Eusébio Conceição

Fabian Sá

Fátima Farinha

Fernando Branco

Fernando Cánovas

Fernando Martins

Flávio Martins

Francisca Segura

Francisco Guzmán Navarro

Francisco Javier Vicario Llerena

Gabriela Schutz

Gil Fraqueza

Giovanna Concu

Gonçalo Prates

Gorete Dinis

Helena Fernandez

Hugo Pinto

Isménio Martins

Jacobo Porteiro

Jeff Camkin

Jessie Melo

João Castro Gomes

João Estevão
Instituto Nacional de Técnica Aeroespacial, Spain

University of Huelva, Spain

University of Salerno, Italy

Universidade do Algarve, Faculdade de Economia, Portugal

Universidade do Algarve, FCT and CINTAL, Portugal

Universidade Federal do Espírito Santo, Brazil

Universidade do Algarve, ISE and CEPAC, Portugal

University of Coimbra, Portugal

Universidad Católica San Antonio de Murcia, Spain

Universidade do Algarve, ISE and CIEO, Portugal

Universidade do Algarve, ISE and CIMA, Portugal

University of Huelva, Spain

University of Malaga, Spain

Universidad de Cádiz, Spain

Universidade do Algarve, ISE and CEOT, Portugal

Universidade do Algarve, ISE and CCMAR, Portugal

University of Cagliari, Italy

Universidade do Algarve, ISE and CEG, Portugal

Instituto Politécnico de Portalegre and GOVCOPP, Portugal

Universidade do Algarve, ISE and CIEO, Portugal

Universidade de Coimbra and CES; Universidade do Algarve, FE, Portugal

Universidade do Algarve, ISE, Portugal

University of Vigo, Spain

CENRM and University of Western Australia, Australia

Universidade do Algarve, ISE and MeditBio, Portugal

University of Beira Interior, Portugal

Universidade do Algarve, ISE and CIMA Portugal 
João M. F. Rodrigues

Jorge de Brito

Jorge Isidoro

Jorge Pereira

José António Monteiro

José Carlos Teixeira

José Luís Argain

José Paulo Monteiro

Juan Jose Salas

Juan Manuel Enrique Gomez

Katherine Flynn

Kinga Krauze

Lahcen Boukhattem

Ludovina Galego

Luís Fialho

Luís Oliveira

Manuel Duarte Pinheiro

Manuela Rosa

Marcos Mateus

Margarida Ribau Teixeira

Margarida Vieira

Maria de Belém Costa

María Martín Morales

Mariana Golumbeanu

Mário Costa

Mário Serafim Nunes
Universidade do Algarve, ISE and LARSyS, Portugal

Instituto Superior Técnico, University of Lisbon, Portugal

Universidade do Algarve, ISE and MARE, Portugal

Universidade do Algarve, ISE and MeditBio, Portugal

Universidade do Algarve, FCT and CEPAC, Portugal

Universidade do Minho, Portugal

Universidade do Algarve, FCT and CIMA, Portugal

Universidade do Algarve, FCT and CERIS, Portugal

Centre for New Water Technologies (CENTA), Spain

University of Huelva, Spain

The European Association for Food SafetySAFE consortium, France

European Regional Centre for Ecohydrology of the Polish Academy of Sciences, Poland

University of Cadi Ayyad, Morocco

Universidade do Algarve, ISE and MeditBio, Portugal

University of Evora, Portugal

Universidade do Algarve, ISE, Portugal

Instituto Superior Técnico, University of Lisbon, Portugal

Universidade do Algarve, ISE and CIEO, Portugal

IST, University of Lisbon and MARETEC, Portugal

Universidade do Algarve, FCT and CENSE, Portugal

Universidade do Algarve, ISE and MeditBio, Portugal

Universidade do Algarve, FCT and MeditBio, Portugal

University of Granada, Spain

Balkan Environmental Association (BEnA), Romania

Instituto Superior Técnico, IDMEC, University of Lisbon, Portugal

INESC Inovação, Lisbon, Portugal 
Marisol Correia

Mercedes Gonzalez Wanguemert

Miguel Angel Ridao

Miguel Reimão Costa

Natividade Vieira

Olfa Ben Said

Patrícia Nunes

Paulo Felisberto

Paulo Relvas

Pedro J. S. Cardoso

Rafaela Matos

Raúl Barros

Rijkelt Beumer

Roberto Lam

Rodrigo Braga Moruzzi

Rui Cruz

Rui Lança

Rute Rocha

Sara Raposo

Senhorinha Teixeira

Sérgio de Jesus

Stephen Thomas

Tomás Figueiredo

Vítor Gonçalves
Universidade do Algarve, ESGHT, Portugal Guatizamar S.L e CCMAR, Portugal

University of Seville, Spain

Universidade do Algarve, FCT and CEPAC, Portugal

University of Porto, Portugal

Bizerta Faculty of Sciences, Carthage University, Tunisia

Universidade do Algarve, ISE and MeditBio, Portugal

Universidade do Algarve, ISE and LarSyS, Portugal

Universidade do Algarve, FCT and CIMA, Portugal

Universidade do Algarve, ISE, and LARSyS, Portugal

Laboratório Nacional de Engenharia Civil, Portugal

Universidade do Algarve, FCT and CIMA, Portugal

Department of Agrotechnology and Food Sciences, Wageningen University and Research, The Netherlands

Universidade do Algarve, ISE and LARSyS, Portugal

Universidade Estadual Paulista, Brazil

Universidade do Algarve, ISE and MeditBio, Portugal

Universidade do Algarve, ISE, Portugal

Universidade do Algarve, ESEC and DESYM, Portugal

Universidade do Algarve, FCT and CIMA, Portugal

Universidade do Minho, Portugal

Universidade do Algarve, FCT and LARSyS, Portugal

OGI Groundwater Specialists Ltd., Durham University, UK

Instituto Politécnico de Bragança and CIMO, Portugal

Universidade dos Açores and CIBIO, Azores, Portugal 


\section{Institutional Support}

Universidade do Algarve

UNESCO, UniTwin, Chair on Ecohydrology: Water for Ecosystems and Societies CIMA-Universidade do Algarve

ITeCons-Instituto de Investigação e Desenvolvimento Tecnológico para a Construção, Energia, Ambiente e Sustentabilidade

Associação Portuguesa de Recursos Hídricos

RISCOS-Associação Portuguesa de Riscos, Prevenção e Segurança

Ordem dos Engenheiros

\section{Sponsors}

Fundação para a Ciência e a Tecnologia (FCT)

Ordem dos Engenheiros da Região Sul

OGI-Groundwater Specialists

Boode-Waterwell Systems

PLATIPUS-Earth Anchoring Systems

Águas do Algarve

Câmara Municipal de Faro

Câmara Municipal de Loulé

CACIAL-Cooperativa Agrícola de Citricultores do Algarve

\section{Media Partner}

Sul Informação 


\section{Contents}

Climate Changes and Environmental Protection

Rainfall Trends in Southern Portugal at Different Time Scales . Maria Manuela Portela, Luis Angel Espinosa, Ticiana Studart, and Martina Zelenakova

Nitrate Reductase Activity in Green Macroalgae as an Environmental Indicator of Temperature and Salinity Changes and Its Implication for Climate Change Projections .

Hélder T. Madeira, Jaime Aníbal, Liliana F. Carvalho, Eduardo Esteves, and Cristina Veiga-Pires

Meteorological Drought Assessment in the Eastern Slovakia

Martina Zelenakova, Tatiana Solakova, Helena Hlavata, Pavol Purcz,

Zuzana Vranayova, and Maria Manuela Portela

Spatial and Meteorological Behaviour of Daily Ozone Air Pollution in the Bay of Algeciras (2010-2015)

Inmaculada Rodríguez, Steffanie Van Roode, José A. Moscoso, Juan J. Ruiz-Aguilar, Francisco Javier Gonzalez-Enrique, and Ignacio J. Turias

Projections of Maximum Tidal Inundation Limits of Estuaries and Lagoons of the South Algarve, Forced by Mean Sea

Level Rise: RCP8.5 Scenario . . . . . . . . . . . . . .
Dissanayake Sampath, Tomasz Boski, Delminda Moura, and Cristina Veiga-Pires

Carbon Balance and Ecosystem Services - Golf Courses

Environmental Performance in Algarve, Portugal

Manuela Moreira da Silva, Duarte Marinho, Gil Fraqueza,

Flávia Carpenter, Bárbara Freitas, Carlos O. da Silva, and Paulo Dias 
Education in Sustainability and Promotion of Scientific-Technical Vocations in Pre-university Students Through the Construction of a Solar Vehicle . . . . . . . . . . . . . . . . . . . . . . . . . 603 Yolanda Ceada-Garrido, Juan Manuel Enrique, Antonio Javier Barragán, Ramón Tirado, and José Manuel Andújar

Mobility Patterns of the Elderly Tourist in Algarve.

Celeste Gameiro, Manuela Rosa, Carlos Sousa, and Ilídio Mestre

Neighborhood Sustainability Assessment: The Case of Casal

Bertone Neighborhood in Rome

Luigi Mundula

OD Mobility Estimation Using Artificial Neural Networks . Luis E. Acosta Sánchez, Javier González-Enrique, J. J. Ruiz-Aguilar, J. A. Moscoso-López, and I. J. Turias

\section{Water for Ecosystem and Society}

Greywater Storage, Treatment and Reuse in Residences Sophia Naara Batista Morato, Zacarias Caetano Vieira, Ellen da Silva Santos, and Ingrid Larissa da Silva Santos

Accuracy/Speed Analysis of Pipe Friction Factor Correlations. Luiz Eduardo Muzzo, Diana Pinho, Luiz Eduardo Melo Lima, and Luís Frölén Ribeiro

Nearly Zero Water Buildings

Carla Pimentel-Rodrigues and Armando Silva-Afonso

High Precision Geomatic Tools for Improving Harvest of Olive

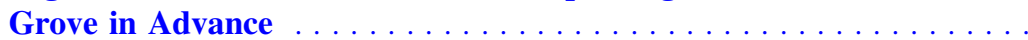
Ma Isabel Ramos, Juan M. Jurado, José L. Cárdenas, Carlos Enríquez, Lidia Ortega, Ma Lourdes Martínez, Francisco R. Feito, and Juan J. Cubillas

Ria Formosa Hydrodynamics and the Best Location for Shellfish Beds

Duarte N. R. Duarte, Della Permata, Manuela Moreira da Silva,

Tiago M. P. Dores, Margarida C. N. Alves, Francisco A. B. Fernandes, Miguel P. dos Santos, and Luís Chicharo

Management Optimization of Waste Water Treatment Plants

Sewage Sludge Produced in the Algarve

Nuno Silva, António Martins, and Jaime Aníbal

Monitoring of Trace Elements on the Anaerobic Digestion

of Saline Sewage.

Lauren N. M. Yabuki and Marcelo L. Garcia 


\title{
Accuracy/Speed Analysis of Pipe Friction Factor Correlations
}

\author{
Luiz Eduardo Muzzo ${ }^{1,2(\bowtie)}$, Diana Pinho ${ }^{3,4}$, Luiz Eduardo Melo Lima ${ }^{5}$, \\ and Luís Frölén Ribeiro ${ }^{6,7}$ \\ 1 Bragança Polytechnic Institute, Bragança, Portugal \\ ${ }^{2}$ Federal University of Technology - Paraná, Ponta Grossa, Brazil \\ luizmuzzo@alunos .utfpr.edu.br \\ 3 Research Centre in Digitalization and Intelligent Robotics (CeDRI) - Bragança \\ Polytechnic Institute, Bragança, Portugal \\ diana@ipb.pt \\ ${ }^{4}$ MEtRICs, Mechanical Engineering Department, University of Minho, \\ Braga, Portugal \\ ${ }^{5}$ Department of Mechanics, Federal University of Technology - Paraná, \\ Ponta Grossa, Brazil \\ lelima@utfpr.edu.br \\ ${ }^{6}$ Mechanical Technical Department, Bragança Polytechnic Institute, \\ Bragança, Portugal \\ frolen@ipb.pt \\ 7 Centre for Renewable Energy Research - INEGI, Porto, Portugal
}

\begin{abstract}
The Colebrook [1] equation is considered the standard for the calculation of friction factor for turbulent flow in commercial pipes, but it is implicit, and therefore it must be computed by iterative methods. Although such iterative computation quickly converges, the computational time in large pipe system simulations can be reduced using an accurate explicit correlation. A review of the up to date literature identified 30 different explicit correlations. In order to determine which correlation is the best alternative to Colebrook's, both accuracy and computational burden were compared. The accuracy of each explicit correlation was compared against Colebrook's correlation using the mean and maximum relative errors and the coefficient of determination. Also, the computational time of each equation was measured using the tic and toc functions in GNU Octave software. It was found that the iterative computation of the Colebrook equation demands about 2.6 times the computational time of the slowest explicit correlation. The correlations with the best balance between accuracy and computational burden are, in decreasing order of accuracy and increasing order of speed, correlations by Serghides [13] (Eqs. (17), (18), (19), and (20)), by Shacham [8] (Eqs. (10) and (11)), by Brkić and Praks [33] (Eqs. (53), (54), (55), and (56)), and by Fang et al. [19] (Eq. (28)).
\end{abstract}

Keywords: Pipe flow $\cdot$ Friction factor $\cdot$ Colebrook $\cdot$ Correlations

(C) Springer Nature Switzerland AG 2020

J. Monteiro et al. (Eds.): INCREaSE 2019, pp. 664-679, 2020.

https://doi.org/10.1007/978-3-030-30938-1_51 


\section{Introduction}

Fluid flow in pipes and ducts is widely used in applications such as the transportation of oil and gas, irrigation, water distribution, air conditioning, and power plants. The friction factor is used in the computation of head loss or pressure drop in pipes, which is the loss of the flow's mechanical energy due to viscous friction. Major or distributed losses are caused by viscous friction caused by the pipe's wall while minor or local losses are caused by the recirculating and turbulent mixture of fluid caused by inlets, outlets, contractions, expansions, curves, valves, junctions, among others structural features of the pipe through the flow's path. These losses $h_{L}$ are defined as:

$$
h_{L}=f \frac{L}{D} \frac{\bar{V}^{2}}{2 g}
$$

where $f$ is the friction factor, $L$ is the pipe's length, $D$ is the pipe's internal diameter, $\bar{V}$ is the average fluid velocity, and $g$ is the gravitational acceleration. For minor losses, an equivalent pipe length can be used.

For laminar flow, the friction factor is computed analytically as a function of the Reynolds number, Re, and for pipes of circular section it is defined as:

$$
f=\frac{64}{\operatorname{Re}}
$$

where Re is defined as:

$$
\operatorname{Re}=\frac{\rho \bar{V} D}{\mu}
$$

where $\rho$ is the fluid's specific mass and $\mu$ is the fluid's dynamic viscosity. For transition and turbulent flow in commercial pipes, Colebrook [1] proposed, in 1939, Eq. (4) based on experimental data.

$$
\frac{1}{\sqrt{f}}=-2 \log _{10}\left(\frac{\epsilon / D}{3.7}+\frac{2.51}{\operatorname{Re} \sqrt{f}}\right)
$$

where $\epsilon$ is the absolute internal roughness of the pipe and $\epsilon / D$ is its relative roughness. Equation (4) is considered the standard in friction factor computation, but it is implicit, and therefore it must be computed by some iterative method. In 1944, Moody [3] plotted this equation in a diagram to simplify its usage in engineering. Also, many explicit correlations were proposed since then, mostly as approximations of Colebrook's implicit correlation. Although the iterative computation converges quickly, computational time in large pipe system simulations can be reduced using a sufficiently accurate explicit correlation.

In this context, the objective of this work is to make a comparative analysis between the Colebrook equation and explicit correlations available in the literature. The accuracy, against the Colebrook equation, and the computational time of the correlations are compared. To check the accuracy of Colebrook's correlation relative to experimental data in real pipes is beyond the objective of this 
work. It should be mentioned that the application of any correlation in real pipe systems depends on measurements of relative roughness and Reynolds number, and therefore they propagate intrinsic uncertainties to the friction factor [2].

\section{Explicit Correlations}

Since Colebrook put forward his equation, many explicit correlations for turbulent friction factor were proposed. Here, the chronological appearance of 30 different explicit equations is depicted from Eq. (5), which was proposed by Moody [4] himself in 1947, to Brkić and Praks' [33] in 2019.

$$
f=0.0055\left[1+\left(2 \times 10^{4} \frac{\epsilon}{D}+\frac{10^{6}}{\operatorname{Re}}\right)^{1 / 3}\right]
$$

In 1966, Wood [5] suggested Eq. (6). It should be noted that this equation is not valid to relative roughness equal to zero.

$$
f=0.53 \frac{\epsilon}{D}+0.094\left(\frac{\epsilon}{D}\right)^{0.225}+88\left(\frac{\epsilon}{D}\right)^{0.44} \operatorname{Re}^{A_{1}}
$$

where:

$$
A_{1}=-1.62\left(\frac{\epsilon}{D}\right)^{0.134}
$$

Equation (8) was proposed by Churchill [6] in 1973.

$$
f=\left\{-2 \log _{10}\left[\frac{\epsilon / D}{3.7}+\left(\frac{7}{\mathrm{Re}}\right)^{0.9}\right]\right\}^{-2}
$$

Swamee and Jain [7] introduced Eq. (9) in 1976.

$$
f=\frac{0.25}{\left\{\log _{10}\left[(\epsilon / D) / 3.7+5.74 / \operatorname{Re}^{0.9}\right]\right\}^{2}}
$$

Equation (10) was recommended by Shacham [8] in 1980.

$$
f=\left\{\left[A_{2}\left(1-\ln A_{2}\right)-\frac{\epsilon / D}{3.7}\right] /\left(1.15129 A_{2}+\frac{2.51}{\operatorname{Re}}\right)\right\}^{-2}
$$

where:

$$
A_{2}=\frac{\epsilon / D}{3.7}-\frac{5.02}{\operatorname{Re}} \log _{10}\left(\frac{\epsilon / D}{3.7}+\frac{14.5}{\operatorname{Re}}\right)
$$

In 1981, Eq. (12) was suggested by Barr [9].

$$
f=\left(-2 \log _{10}\left\{\frac{\epsilon / D}{3.7}+\frac{4.518 \log _{10}(\operatorname{Re} / 7)}{\operatorname{Re}\left[1+\left(\operatorname{Re}^{0.52} / 29\right)(\epsilon / D)^{0.7}\right]}\right\}\right)^{-2}
$$


Zigrang and Sylvester [10] proposed, in 1982, Eq. (13).

$$
f=\left\{-2 \log _{10}\left[\frac{\epsilon / D}{3.7}-\frac{5.02}{\operatorname{Re}} \log _{10}\left(\frac{\epsilon / D}{3.7}-\frac{5.02}{\operatorname{Re}} \log _{10} A_{3}\right)\right]\right\}^{-2}
$$

where:

$$
A_{3}=\frac{\epsilon / D}{3.7}+\frac{13}{\operatorname{Re}}
$$

In 1983, Haaland [11] submitted Eq. (15).

$$
f=\left\{-1.8 \log _{10}\left[\frac{6,9}{\operatorname{Re}}+\left(\frac{\epsilon / D}{3,7}\right)^{1.11}\right]\right\}^{-2}
$$

In 1984, Chen [12] recommended Eq. (16).

$$
f=0.3164\left(\frac{1}{\operatorname{Re}^{0.83}}+0.11 \frac{\epsilon}{D}\right)^{0.3}
$$

Equation (17) was proposed by Serghides [13] in 1984.

$$
f=\left[A_{4}-\frac{\left(B_{4}-A_{4}\right)^{2}}{A_{4}-2 B_{4}+C_{4}}\right]^{-2}
$$

where:

$$
\begin{gathered}
A_{4}=-2 \log _{10}\left(\frac{\epsilon / D}{3.7}+\frac{12}{\operatorname{Re}}\right) \\
B_{4}=-2 \log _{10}\left(\frac{\epsilon / D}{3.7}+\frac{2.51 A_{4}}{\operatorname{Re}}\right) \\
C_{4}=-2 \log _{10}\left(\frac{\epsilon / D}{3.7}+\frac{2.51 A_{4}}{\operatorname{Re}}\right)
\end{gathered}
$$

In 1997, Manadilli [14] presented Eq. (21).

$$
f=\left[-2 \log _{10}\left(\frac{\epsilon / D}{3.70}+\frac{95}{\operatorname{Re}^{0.983}}-\frac{96.82}{\mathrm{Re}}\right)\right]^{-2}
$$

Sonnad and Goudar [15], in 2006, proposed Eq. (22).

$$
f=\left\{0.8686 \ln \left[\frac{0.4587 \mathrm{Re}}{A_{5}^{A_{5} /\left(A_{5}+1\right)}}\right]\right\}^{-2}
$$

where:

$$
A_{5}=0.124 \frac{\epsilon}{D} \operatorname{Re}+\ln (0.4587 \mathrm{Re})
$$


Vatankhah [16], in 2014, optimized Eq. (22). Equation (24) is the optimized form.

$$
f=\left\{0.8686 \ln \left[\frac{0.4599 \mathrm{Re}}{\left(A_{6}-0.2753\right)^{A_{6} /\left(A_{6}+0.9741\right)}}\right]\right\}
$$

where:

$$
A_{6}=0.124 \frac{\epsilon}{D} \operatorname{Re}+\ln (0.4599 \operatorname{Re})
$$

Avci and Karagoz [17] suggested, in 2009, Eq. (26).

$$
f=\frac{6.4}{\{\ln \operatorname{Re}-\ln [1+0.01 \operatorname{Re}(\epsilon / D)(1+10 \sqrt{\epsilon / D})]\}^{2.4}}
$$

Equation (27) was introduced by Papaevangelou et al. [18] in 2010.

$$
f=\frac{0.2479-0.0000947\left(7-\log _{10} \operatorname{Re}\right)^{4}}{\left\{\log _{10}\left[(\epsilon / D) / 3.615+7.366 / \operatorname{Re}^{0.9142}\right]\right\}^{2}}
$$

In 2011, Fang et al. [19] recommended Eq. (28).

$$
f=1.613\left\{\ln \left[0.234\left(\frac{\epsilon}{D}\right)^{1.1007}-\frac{60.525}{\operatorname{Re}^{1.1105}}+\frac{56.291}{\operatorname{Re}^{1.0712}}\right]\right\}^{-2}
$$

Ghanbari et al. [20] proposed, in 2011, Eq. (29).

$$
f=\left\{-1.52 \log _{10}\left[\left(\frac{\epsilon / D}{7.21}\right)^{1.042}+\left(\frac{2.731}{\operatorname{Re}}\right)^{0.9152}\right]\right\}^{-2.169}
$$

Equation (30) was submitted by Samadianfard [21] in 2012.

$$
f=\left[\frac{\operatorname{Re}^{\epsilon / D}-0.6315093}{\operatorname{Re}^{1 / 3}+\operatorname{Re}(\epsilon / D)}\right]+0.0275308\left(\frac{6.929841}{\operatorname{Re}}+\epsilon / D\right)^{1 / 9}+A_{7}
$$

where:

$$
A_{7}=\left[\frac{10^{\epsilon / D}}{(\epsilon / D)+4.781616}\right]\left(\sqrt{\epsilon / D}+\frac{9.99701}{\operatorname{Re}}\right)
$$

In 2014, Vatankhah [16] also proposed Eq. (32).

$$
f=\left[\frac{2.51 / \operatorname{Re}+1.1513 A_{8}}{A_{8}-(\epsilon / D) / 3.71-2.3026 A_{8} \log _{10}\left(A_{8}\right)}\right]^{2}
$$

where: 


$$
A_{8}=\frac{6.0173}{\operatorname{Re}\left[0.07(\epsilon / D)+\mathrm{Re}^{-0.885}\right]^{0.109}}+\frac{\epsilon / D}{3.71}
$$

In 2015, Heydari et al. [22] proposed Eq. (34).

$$
f=\left[0.42+16.27 \frac{\epsilon}{D}-1.81 \log _{10}\left(\frac{\epsilon / D}{3.7}+\frac{5.74}{\operatorname{Re}^{0.9}}\right)+A_{9}+B_{9}\right]^{-2}
$$

where:

$$
\begin{gathered}
A_{9}=-54.81(\epsilon / D)^{2}+0.02\left[\log _{10}\left(\frac{\epsilon / D}{3.7}+\frac{5.74}{\mathrm{Re}^{0.9}}\right)\right]^{2} \\
B_{9}=8.74(\epsilon / D) \log _{10}\left(\frac{\epsilon / D}{3.7}+\frac{5.74}{\mathrm{Re}^{0.9}}\right)
\end{gathered}
$$

Equation (37) was suggested by Offor and Alabi [23] in 2016.

$$
f=\left(-2 \log _{10}\left\{\frac{\epsilon / D}{3.71}-\frac{1.975}{\operatorname{Re}} \ln \left[\left(\frac{\epsilon / D}{3.93}\right)^{1.092}+\frac{7.627}{\operatorname{Re}+395.9}\right]\right\}\right)^{-2}
$$

In 2016, Beluco and Schettini [24] presented Eq. (38).

$$
f=0.3009\left\{\log _{10}\left[\left(\frac{\epsilon / D}{3.7315}\right)^{1.0954}+\left(\frac{5.9802}{\operatorname{Re}}\right)^{0.9695}\right]\right\}^{-2}
$$

In 2017, Biberg [25] recommended Eq. (39).

$$
f=\left\{\frac{2}{\ln 10}\left[\ln \left(\frac{\ln 10 \mathrm{Re}}{5.02}\right)+\left(\frac{1}{A_{10}}-1\right) \ln A_{10}\right]\right\}^{-2}
$$

where:

$$
A_{10}=\ln \left(\frac{\ln 10 \mathrm{Re}}{5.02}\right)+\frac{\ln 10 \mathrm{Re}}{18.574} \frac{\epsilon}{D}
$$

In 2017, Brkić and Ćojbašić [26] suggested optimized forms of various explicit correlations. Their goal was to minimize the maximum relative error of these equations. Equation (41) is the optimized form of the correlation proposed by Eck [27] in 1966 and Eq. (42) is the optimized form of the correlation introduced by Chen [28] in 1979.

$$
f=\left[-1.963 \log _{10}\left(\frac{14.064}{\operatorname{Re}}+\frac{\epsilon / D}{4.034}\right)\right]^{-2}
$$




$$
f=\left(-2.003 \log _{10}\left\{\frac{\epsilon / D}{3.689}-\frac{4.933}{\operatorname{Re}} \log _{10}\left[\frac{1}{2.762}\left(\frac{\epsilon}{D}\right)^{1.109}+A_{11}\right]\right\}\right)^{-2}
$$

where:

$$
A_{11}=\frac{5.89}{\operatorname{Re}^{0.923}}
$$

Equation (44) is the optimized form of Round's [29] equation from 1980.

$$
f=\left\{1.898 \log _{10}\left[\frac{\operatorname{Re}}{0.202 \operatorname{Re}(\epsilon / D)+9.779}\right]\right\}^{-2}
$$

Equation (45) is the optimized form of correlation by Romeo et al. [30] from 2002.

$$
f=\left\{-2 \log _{10}\left[\frac{\epsilon / D}{3.7106}-\frac{5}{\operatorname{Re}} \log _{10}\left(\frac{\epsilon / D}{3.8597}-\frac{4.795}{\operatorname{Re}} \log _{10} A_{12}\right)\right]\right\}^{-2}
$$

where:

$$
A_{12}=\left(\frac{\epsilon / D}{7.646}\right)^{0.9685}+\left(\frac{4.9755}{206.2795+\mathrm{Re}}\right)^{0.0,8759}
$$

Equation (47) is the optimized form of the equation proposed by Buzzelli [31] in 2008.

$$
f=\left\{A_{13}-\left[\frac{A_{13}+1.9999 \log _{10}\left(B_{13} / \mathrm{Re}\right)}{0.9996+2.1018 / B_{13}}\right]\right\}^{-2}
$$

where:

$$
\begin{aligned}
& A_{13}=\frac{0.7314 \ln \operatorname{Re}-1.3163}{1.0025+1.2435 \sqrt{\epsilon / D}} \\
& B_{13}=\frac{\epsilon / D}{3.7165} \operatorname{Re}+2.5137 A_{13}
\end{aligned}
$$

Equation (50) was introduced by Gregory and McEnery [32] in 2017.

$$
f=\left[-2 \log _{10}\left(\frac{\epsilon / D}{3.7}+\frac{1.64 A_{14}}{\operatorname{Re}^{B_{14}}}\right)\right]^{-2}
$$

where:

$$
\begin{aligned}
& A_{14}=0.9-4.9 \frac{\epsilon}{D}+0.1 e^{-400(\epsilon / D)} \\
& B_{14}=1-\frac{1}{4+0.208 \ln (\operatorname{Re} / 3000)}
\end{aligned}
$$


In 2019, Brkić and Praks [33] submitted Eq. (53).

$$
f=\left\{0.8686\left[A_{15}-C_{15}+\frac{C_{15}}{A_{15}+B_{15}}\right]\right\}^{-2}
$$

where:

$$
\begin{gathered}
A_{15}=\ln \operatorname{Re}-0.779397488 \\
B_{15}=\frac{\operatorname{Re}(\epsilon / D)}{8.0878} \\
C_{15}=\ln \left(A_{15}+B_{15}\right)
\end{gathered}
$$

This list is not exhaustive, but it contains the explicit correlations that are frequently referenced in similar accuracy and complexity comparison works [3438]. Some correlations were excluded because the required reference was not available, due to minor changes from the presented ones, or due to the excessive complexity of their equations.

\section{Methodology}

The evaluation of accuracy, against the Colebrook equation, and computational burden of the explicit correlations presented in Sect. 2 were performed in GNU Octave software, version 4.2.1, running in a computer with an Intel Core i5-6600 $3.90 \mathrm{GHz}$ processor and $8 \mathrm{~GB}$ of RAM.

\subsection{Accuracy Evaluation}

The accuracy of the 30 explicit correlations from Eqs. (5) to (56) was evaluated by their range of error (minimum and maximum errors), average error and coefficient of determination or $R^{2}$, all against the Colebrook equation.

A domain of 402 values of relative roughness in $10^{-6} \leq \epsilon / D \leq 0.05$ plus $\epsilon / D=0\left(0,1.0 \times 10^{-6}, 1.1 \times 10^{-6}, \ldots, 9.9 \times 10^{-6}, 1.0 \times 10^{-5}, 1.1 \times 10^{-5}, \ldots\right.$, $\left.5.0 \times 10^{-2}\right)$ by 4201 values of Reynolds number in $4000 \leq \operatorname{Re} \leq 10^{8}\left(4.00 \times 10^{3}\right.$, $\left.4.01 \times 10^{3}, \ldots, 9.99 \times 10^{3}, 1.00 \times 10^{4}, 1.01 \times 10^{4}, \ldots, 1.00 \times 10^{8}\right)$ was created yielding a total of 1688802 points, corresponding to the ranges of values used by Moody [3] in his diagram. The friction factor was calculated for all points by the Colebrook equation and by each explicit correlation. Smooth pipe, i.e. $\epsilon / D=0$, was not evaluated in Wood's [5] correlation (Eqs. (6) and (7)) because it does not accept this value. The Colebrook equation was calculated by the fixed point iterative method defined as:

$$
\frac{1}{\sqrt{f_{i+1}}}=-2 \log _{10}\left(\frac{\epsilon / D}{3.7}+\frac{2.51}{\operatorname{Re} \sqrt{f_{i}}}\right), i=1,2, \ldots, N
$$


where $f_{i}$ is the friction factor in the iteration $i, f_{i+1}$ is the friction factor to be used in the next iteration, and $N$ is the number of iterations. An initial guess is used for $f_{i}$ in the first iteration, and it is followed by the iterations until the criterion of convergence is achieved. The initial value used was 0.0425 , which is the average of the minimum and maximum friction factor values in the Moody diagram, i.e. 0.005 and 0.08 . The criterion of convergence was an absolute error between two consecutive iterations of less than $10^{-10}$. Using this iterative method, Colebrook's correlation converges after an average of 8 iterations. Also, the initial value does not affect too much the convergence as long as it is larger than zero.

The relative dimensionless error $\varepsilon$ of each point is defined as:

$$
\varepsilon=\frac{f_{C}-f_{C W}}{f_{C W}}
$$

where $f_{C}$ is the friction factor calculated by the correspondent explicit correlation and $f_{C W}$ is the friction factor calculated by the Colebrook equation. The average of the relative error $\bar{\varepsilon}$ is defined as:

$$
\bar{\varepsilon}=\frac{1}{P} \sum_{i=1}^{P} \frac{\left|f_{C_{i}}-f_{C W_{i}}\right|}{f_{C W_{i}}}
$$

where $P$ is the number of points used in the accuracy evaluation, i.e. 1688802, $f_{C_{i}}$ is the friction factor calculated by the correspondent explicit correlation for the point, and $f_{C W_{i}}$ is the friction factor calculated by Colebrook's correlation for the point. Also, the ratio of maximum relative error was calculated as the correlation's maximum relative error divided by the smallest maximum relative error among all correlations.

The coefficient determination $R^{2}$ is defined as:

$$
R^{2}=1-\sum_{i=1}^{P}\left(f_{C W_{i}}-f_{C_{i}}\right)^{2} / \sum_{i=1}^{P}\left(f_{C W_{i}}-\bar{f}_{C W}\right)^{2}
$$

where $\bar{f}_{C W}$ is the average of all values of friction factor calculated by the Colebrook equation, which is approximately 0.025 , defined as:

$$
\bar{f}_{C W}=\frac{1}{P} \sum_{i=1}^{P} f_{C W_{i}}
$$

\subsection{Computational Burden Evaluation}

The computational times of the 30 explicit correlations from Eqs. (5) to (56) and of the Colebrook equation were measured using the tic and toc functions in GNU Octave software release 4.2.1.

It was used a domain of 42 values of relative roughness in $10^{-6} \leq \epsilon / D \leq 0.05$ plus $\epsilon / D=0$ by 421 values o Reynolds number in $4000 \leq \operatorname{Re} \leq 10^{8}$ with a total 
of 17682 points. The friction factor was calculated by the Colebrook equation and by each explicit correlation for all points, and the time taken was measured. As in the accuracy evaluation, smooth pipe, i.e. $\epsilon / D=0$, was not evaluated in Eqs. (6) and (7), which correspond to the correlation proposed by Wood [5]. Also, the Colebrook equation was calculated by the fixed point iterative method with 0.0425 as an initial value and an absolute error between two consecutive iterations of less than $10^{-10}$ was used as the criterion of convergence. This computational time measurement was repeated nine times and were calculate their average and standard deviation $\sigma$ :

$$
\sigma=\sqrt{\frac{1}{M-1} \sum_{i=1}^{M}\left(t_{i}-\bar{t}\right)^{2}}
$$

where $M$ is the number of measurements, i.e. $9, t_{i}$ is the computational time measured and $\bar{t}$ is the average of the measured computational times. Also, in order to make this computational time dimensionless and independent of the computational power of the computer, the ratio of computational time was calculated as the correlation's computational time divided by the smallest computational time among all correlations.

\section{Results and Discussion}

The results from both accuracy and computational time evaluations are summarized and discussed in this section. The computational codes developed in this work are published in the Code Ocean platform and available in: https:// codeocean.com/capsule/7657118/.

\subsection{Accuracy of the Correlations}

Table 1 shows the accuracy of the explicit correlations. Their ratio of maximum error, range of error, average error and $R^{2}$ are shown. All 30 explicit correlations are ranked from the smallest to the largest maximum relative error, in absolute values. The ranking does not change significantly if the average error is used as the main criteria of comparison.

Correlation by Serghides [13] (Eqs. (17) to (20)) is the most accurate one, with maximum relative error of $0.00314 \%$. The next is correlation by Shacham [8] (Eqs. (10) and (11)), with maximum relative error of $0.01740 \%$. With maximum relative errors between $0.10407 \%$ and $0.15285 \%$ are correlations by Buzzelli [26] (Eqs. (47) to (49)), by Zigrang and Sylvester [10] (Eqs. (13) and (14)), by Brkić and Praks [33] (Eqs. (53) to (56)), by Offor and Alabi [23] (Eq. (37)), by Vatankhah [16] (Eqs. (32) and (33)), by Romeo et al. [26] (Eqs. (45) and (46)), by Sonnad and Goudar [16] (Eqs. (24) and (25)) and by Biberg [25] (Eqs. (39) and $(40))$. All these correlations have average error equal or less than $0.05500 \%$ and $R^{2}$ equal to 1.00000 . 
Table 1. Ranking of the most to the less accurate explicit correlations. Error ratio is the maximum error of the correlation divided by the smallest maximum error among all correlations, $\varepsilon$ is the range of errors, $\bar{\varepsilon}$ is the average error in absolute value and $R^{2}$ is the coefficient of determination.

\begin{tabular}{|c|c|c|c|c|}
\hline Equation(s) & Error ratio & $\varepsilon[\%]$ & $\bar{\varepsilon}[\%]$ & $R^{2}$ \\
\hline$(17),(18),(19)$, and $(20)$ & 1.00000 & {$[-0.00314,0.00000]$} & 0.00055 & 1.00000 \\
\hline (10) and (11) & 5.54637 & {$[-0.00044,0.01740]$} & 0.00206 & 1.00000 \\
\hline$(47),(48)$, and $(49)$ & 33.16356 & {$[-0.10407,0.07123]$} & 0.03280 & 1.00000 \\
\hline (13) and (14) & 36.20211 & {$[-0.11360,0.04060]$} & 0.02447 & 1.00000 \\
\hline$(53),(54),(55)$, and $(56)$ & 39.66605 & {$[-0.12447,0.08703]$} & 0.05500 & 1.00000 \\
\hline$(37)$ & 39.93023 & {$[-0.12530,0.04924]$} & 0.05199 & 1.00000 \\
\hline$(32)$ and (33) & 39.93048 & {$[-0.12530,0.05958]$} & 0.04297 & 1.00000 \\
\hline$(45)$ and (46) & 42.31998 & {$[-0.13280,0.00137]$} & 0.04521 & 1.00000 \\
\hline (24) and (25) & 44.33863 & {$[-0.13913,-0.00509]$} & 0.04811 & 1.00000 \\
\hline (39) and (40) & 48.71029 & {$[-0.10478,0.15285]$} & 0.03583 & 1.00000 \\
\hline (42) and (43) & 96.82851 & {$[-0.30384,0.10366]$} & 0.12043 & 0.99999 \\
\hline$(28)$ & 156.62707 & {$[-0.44092,0.49149]$} & 0.16294 & 0.99999 \\
\hline (12) & 170.10547 & {$[-0.53378,0.32094]$} & 0.06569 & 0.99999 \\
\hline$(27)$ & 257.77584 & {$[-0.80889,0.57827]$} & 0.17521 & 0.99998 \\
\hline$(22)$ and $(23)$ & 316.34152 & {$[0.00187,0.99267]$} & 0.25125 & 0.99993 \\
\hline$(34),(35)$, and $(36)$ & 417.81326 & {$[-1.25486,1.31108]$} & 0.67725 & 0.99984 \\
\hline$(50),(51)$, and $(52)$ & 431.85845 & {$[-1.35515,0.72541]$} & 0.22497 & 0.99997 \\
\hline$(15)$ & 453.68782 & {$[-1.42365,1.31384]$} & 0.44954 & 0.99991 \\
\hline$(21)$ & 869.52101 & {$[-0.00407,2.72852]$} & 0.38169 & 0.99972 \\
\hline$(29)$ & 922.96631 & {$[-2.89623,2.15521]$} & 0.72309 & 0.99937 \\
\hline$(26)$ & 954.27655 & {$[-2.99448,2.90109]$} & 1.03591 & 0.99901 \\
\hline$(38)$ & 1046.80961 & {$[-0.93384,3.28485]$} & 0.28874 & 0.99997 \\
\hline (9) & 1070.18734 & {$[-0.70862,3.35820]$} & 0.51644 & 0.99959 \\
\hline$(8)$ & 1089.57825 & {$[-0.62086,3.41905]$} & 0.52977 & 0.99957 \\
\hline$(44)$ & 1742.94614 & {$[-5.15812,5.46929]$} & 2.31334 & 0.99863 \\
\hline$(41)$ & 2545.82949 & {$[-6.94966,7.98871]$} & 2.11387 & 0.99685 \\
\hline (30) and (31) & 3956.45491 & {$[-12.41519,7.42849]$} & 1.75428 & 0.99889 \\
\hline$(5)$ & 5066.56332 & {$[-15.89867,12.53223]$} & 3.09869 & 0.97956 \\
\hline (6) and (7) & 8997.41867 & {$[-28.23353,6.24061]$} & 3.30019 & 0.99387 \\
\hline$(16)$ & 14578.89625 & {$[-45.74798,10.37852]$} & 8.63559 & 0.98286 \\
\hline
\end{tabular}


Correlations by Chen [26] (Eq. (43)), by Fang et al. [19] (Eq. (28)), by Barr [9] (Eq. (12)), by Papaevangelou et al. [18] (Eq. (27)) and by Sonnad and Goudar [15] (Eqs. (22) and (23)) have maximum relative errors between $0.30384 \%$ and $0.99267 \%$. The correlations by Heydari et al. [22] (Eqs. (34) and (35)), by Gregory and McEnery [32] (Eqs. (50) to (52)), and by Haaland [11] (Eq. (15)) have, respectively, $1.31108 \%, 1.35515 \%$, and $1.42365 \%$ maximum relative errors.

Correlations by Manadilli [14] (Eq. (21)), by Ghanbari et al. [20] (Eq. (29)), by Avci and Karagoz [17] (Eq. (26)), by Beluco and Schettini [24] (Eq. (38)), by Swamee and Jain [7] (Eq. (9)), by Churchill [6] (Eq. (8)), and by Round [26] (Eq. (44)) have maximum relative errors between $2.72852 \%$ and $5.46929 \%$.

With maximum relative error equal or greater than $7.98871 \%$ are correlations by Eck [26] (Eq. (41)), by Samadianfard [21] (Eqs. (30) and (31)), by Moody [4] (Eq. (5)), by Wood [5] (Eqs. (6) and (7)) and by Chen [12] (Eq. (16)). Among the explicit correlations analysed, equation by Chen has the greatest maximum relative error of $45.74798 \%$, the greatest average relative error of $8.63559 \%$ and the least $R^{2}$ of 0.98286 .

\subsection{Computational Burden of the Correlations}

Table 2 shows the computational time of the explicit and Colebrook's correlations. Their time ratio, average and standard deviation of computational time are presented. All correlations are ranked from the smallest to the largest average computational time.

As expected, in general, the fastest correlations are also the least accurate ones. The most accurate explicit correlation (Eqs. (17) to (18)) has computational time 4.5 times greater than the fastest (Eq. (5)). One important result is that the fixed point iterative method of calculation of the Colebrook equation, which is Eq. (57), is the most time consuming one. Its computational time is 2.6 times longer than the slowest explicit correlation (Eqs. (34) to (36)).

\subsection{Speed/Accuracy Analysis of the Correlations}

The computational time of Colebrook's correlation is larger than those of the explicit correlations. Therefore, depending on the accuracy need, any explicit correlation can be used to reduce the computational time in simulations. Figure 1 shows the plot of the explicit correlations' ratio of maximum relative error (in the abscissa axis) by their ratio of computational time (in the ordinate axis) and allows to determine the explicit correlations with the best balance between accuracy and computational burden.

The points marked with squares are the correlations with the best balance between accuracy and computational time. The shorter computational time and lower maximum relative error, the better. Points closer to the origin $(0,0)$ or to either axis mean better explicit correlations. The first one, in the top left, is Serghides' [13] correlation (Eqs. (17) to (20)), with maximum error of $0.00314 \%$ and ratio of time of 4.47833. Its more accurate and less time consuming than 
Table 2. Ranking of the fastest to the slowest correlations. Time ratio is the average computational time of the correlation divided by the smallest average computational time among all correlations, $\bar{t}$ is the average computational time and $\sigma$ is its standard deviation.

\begin{tabular}{|c|c|c|c|}
\hline Equation(s) & Time ratio & $\bar{t}[\mathrm{~s}]$ & $\sigma[\mathrm{s}]$ \\
\hline (5) & 1.00000 & 0.23821 & 0.00438 \\
\hline (16) & 1.02485 & 0.24413 & 0.01804 \\
\hline$(28)$ & 1.62218 & 0.38641 & 0.00509 \\
\hline (8) & 1.62941 & 0.38814 & 0.00686 \\
\hline$(41)$ & 1.65171 & 0.39345 & 0.02216 \\
\hline (9) & 1.66194 & 0.39589 & 0.01599 \\
\hline$(38)$ & 1.66650 & 0.39697 & 0.01020 \\
\hline$(15)$ & 1.70599 & 0.40638 & 0.02625 \\
\hline$(29)$ & 1.80823 & 0.43074 & 0.04267 \\
\hline$(44)$ & 2.01129 & 0.47910 & 0.05840 \\
\hline$(21)$ & 2.07453 & 0.49417 & 0.05694 \\
\hline$(53),(54),(55)$, and $(56)$ & 2.19184 & 0.52211 & 0.00555 \\
\hline (6) and (7) & 2.19406 & 0.52264 & 0.00486 \\
\hline (22) and (23) & 2.31085 & 0.55046 & 0.00441 \\
\hline (24) and (25) & 2.33074 & 0.55520 & 0.00871 \\
\hline$(37)$ & 2.56558 & 0.61114 & 0.01196 \\
\hline (26) & 2.64305 & 0.62959 & 0.08425 \\
\hline$(27)$ & 2.87608 & 0.68510 & 0.07418 \\
\hline (32) and (33) & 3.01924 & 0.71921 & 0.00634 \\
\hline (42) and (43) & 3.05794 & 0.72842 & 0.03721 \\
\hline$(50),(51)$, and $(52)$ & 3.20991 & 0.76462 & 0.00825 \\
\hline$(12)$ & 3.47848 & 0.82869 & 0.06868 \\
\hline$(47),(48)$, and $(49)$ & 3.62546 & 0.86361 & 0.08607 \\
\hline (10) and (11) & 3.62941 & 0.86455 & 0.07591 \\
\hline (39) and (40) & 3.85815 & 0.91904 & 0.03053 \\
\hline (30) and (31) & 3.99840 & 0.95245 & 0.00846 \\
\hline (13) and (14) & 4.00288 & 0.95352 & 0.01309 \\
\hline (45) and (46) & 4.09536 & 0.97555 & 0.00474 \\
\hline$(17),(18),(19)$, and $(20)$ & 4.47833 & 1.06677 & 0.02110 \\
\hline$(34),(35)$, and $(36)$ & 5.16117 & 1.22943 & 0.01771 \\
\hline$(57)$ & 13.33385 & 3,17622 & 0.02452 \\
\hline
\end{tabular}




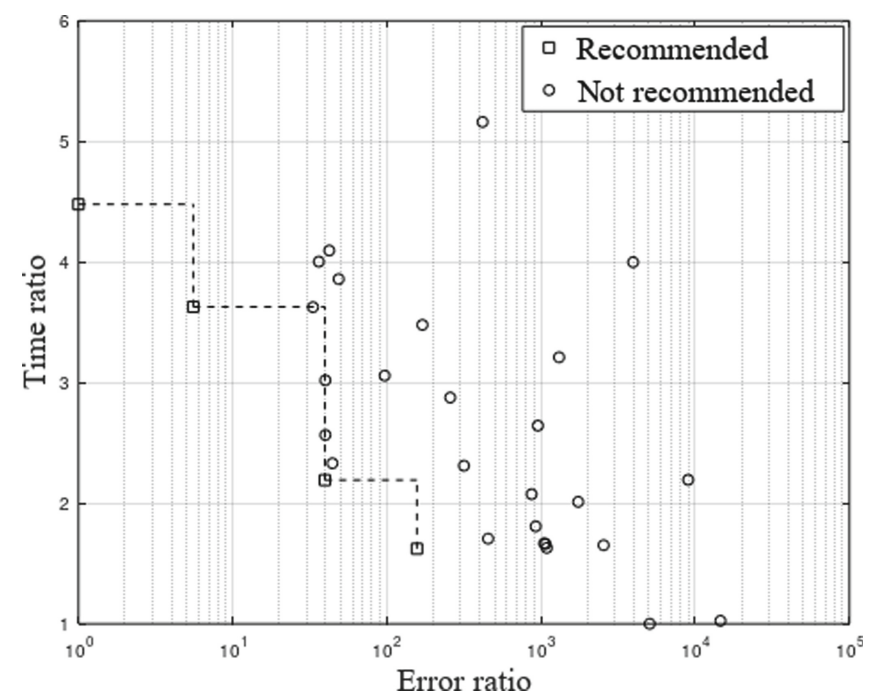

Fig. 1. Ratio of average computational time plotted against ratio of maximum relative error of the explicit correlations. The recommended explicit correlations are those with the best balance between accuracy and computational burden.

the correlation by Heidary et al. [22] (Eqs. (34) and (35)), which is the point in the top of Fig. 1.

The second recommendation is correlation by Shacham [8] (Eqs. (10) and (11)), with maximum relative error of $0.01740 \%$ and ratio of time of 3.62941 . Buzzelli's [26] correlation (Eqs. (47) to (49)) have a very similar ratio of time of 3.62546 , but its maximum relative error is $0.10407 \%$, which is six times greater than Shacham's.

The correlation proposed by Brkić and Praks [33] (Eqs. (53) to (56)) is the third recommendation, with maximum relative error of $0.12447 \%$ and ratio of time of 2.19406. With similar maximum error, but with slightly larger computational time, are correlations by Sonnad and Goudar [16] (Eqs. (24) and (25)), by Offor and Alabi [23] (Eq. (37)), and by Vatankhah [16] (Eqs. (32) and (33)) (on the dotted line).

The fourth and last recommendation is the correlation developed by Fang et al. [19] (Eq. (28)), with a maximum relative error of $0.49149 \%$ and ratio of time of 1.62218. Their correlation consumes about the same computational time as correlation by Haaland [11] (Eq. (15)), which has the ratio of time of 1.70599, but the relative error of the latter is up to $1.42365 \%$. Haaland's equation is the most accurate correlation with only one input of both relative roughness and Reynolds number, and therefore it is the best suited for calculations by hand. The fastest correlation, which is the circle in the bottom of Fig. 1, has error up to $15.89867 \%$, relative to Colebrook's correlation. 


\section{Conclusions}

The use of explicit correlations to calculated the friction factor in pipes reduces the computational time in simulations, compared to the implicit correlation of Colebrook. The slowest correlation is 2.6 times faster than Colebrook's iterative calculation for accuracy of ten decimal places. In decreasing order of accuracy and increasing order of speed, the recommend explicit correlations are: Serghides' [13] (Eqs. (17), (18), (19), and (20)) for maximum error of $0.00314 \%$ and average error of 0.00055\%, Shacham's [8] (Eqs. (10) and (11)) for maximum of $0.01740 \%$ and average of $0.00206 \%$, Brkić and Praks' [33] (Eqs. (53), (54), (55), and (56)) for maximum of $0.12477 \%$ and average $0.05500 \%$, and Fang et al.'s [19] (Eq. (28)) for maximum error of $0.49149 \%$ and average error of $0.16294 \%$.

\section{References}

1. Colebrook, C.F.: Turbulent flow in pipes, with particular reference to the transition region between the smooth and rough pipe laws. J. Inst. Civ. Eng. 11(4), 133-156 (1939)

2. Lira, I.: On the uncertainties stemming from use of the Colebrook-White equation. Ind. Eng. Chem. Res. 52(22), 7550-7555 (2013)

3. Moody, L.F.: Friction factors for pipe flow. Trans. Am. Soc. Mech. Eng. 66, 671$684(1944)$

4. Moody, L.F.: An approximate formula for pipe friction factors. Trans. Am. Soc. Mech. Eng. 69(12), 1005-1011 (1947)

5. Wood, D.J.: An explicit friction factor relationship. Civ. Eng. 36(12), 60-61 (1966)

6. Churchill, S.W.: Empirical expressions for the shear stress in turbulent flow in commercial pipe. AIChE J. 19(2), 375-376 (1973)

7. Swamee, P.K., Jain, A.K.: Explicit equations for pipe-flow problems. J. Hydraul. Div. 102(5), 657-664 (1976)

8. Shacham, M.: Comments on: "An explicit equation for friction factor in pipe". Ind. Eng. Chem. Fundam. 19(2), 228 (1980)

9. Barr, D.I.H.: Solutions of the Colebrook-White function for resistance to uniform turbulent flow. Proc. Inst. Civ. Eng. 71(2), 529-535 (1981)

10. Zigrang, D.J., Sylvester, N.D.: Explicit approximations to the solution of Colebrook's friction factor equation. AIChE J. 28, 514-515 (1982)

11. Haaland, S.E.: Simple and explicit formulas for the friction factor in turbulent pipe flow. J. Fluids Eng. 105(1), 89-90 (1983)

12. Chen, J.J.J.: A simple explicit formula for the estimation of pipe friction factor. Proc. Inst. Civ. Eng. 77(1), 49-55 (1984)

13. Serghides, T.K.: Estimate friction factor accurately. Chem. Eng. 91, 63-64 (1984)

14. Manadilli, G.: Replace implicit equations with signomial functions. Chem. Eng. J. 104(8), 129-130 (1997)

15. Sonnad, J.R., Goudar, C.T.: Turbulent flow friction factor calculation using a mathematically exact alternative to the Colebrook-White equation. J. Hydraul. Eng. 132(8), 863-867 (2006)

16. Vatankhah, A.R.: Comment on "gene expression programming analysis of implicit Colebrook-White equation in turbulent flow friction factor calculation". J. Petrol. Sci. Eng. 124, 402-405 (2014) 
17. Avci, A., Karagoz, I.: A novel explicit equation for friction factor in smooth and rough pipes. J. Fluids Eng. 131(6), 061203 (2009)

18. Papaevangelou, G., Evangelides, C., Tzimopoulos, C.: A new explicit relation for the friction coefficient in the Darcy-Weisbach equation. Prot. Restor. Environ. 166, $1-7(2010)$

19. Fang, X., Xu, Y., Zhou, Z.: New correlations of single-phase friction factor for turbulent pipe flow and evaluation of existing single-phase friction factor correlations. Nucl. Eng. Des. 241(3), 897-902 (2011)

20. Ghanbari, A., Farshad, F.F., Rieke, H.H.: Newly developed friction factor correlation for pipe flow and flow assurance. J. Chem. Eng. Mater. Sci. 2(6), 83-86 (2011)

21. Samadianfard, S.: Gene expression programming analysis of implicit ColebrookWhite equation in turbulent flow friction factor calculation. J. Petrol. Sci. Eng. 92-93, 48-55 (2012)

22. Heydari, A., Narimani, E., Paknniya, F.: Explicit determinations of the Colebrook equation for the flow friction factor by statistical analysis. Chem. Eng. Technol. 38(8), 1387-1396 (2015)

23. Offor, U.H., Alabi, S.B.: An accurate and computationally efficient explicit friction factor model. Adv. Chem. Eng. Sci. 6(03), 237-245 (2016)

24. Beluco, A., Schettini, E.B.C.: An improved expression for a classical type of explicit approximation of the Colebrook White equation with only one internal iteration. Int. J. Hydraul. Eng. 5(1), 19-23 (2016)

25. Biberg, D.: Fast and accurate approximations for the Colebrook equation. J. Fluids Eng. 139(3), 031401 (2017)

26. Brkić, D., Cojbašić, Z.: Evolutionary optimization of Colebrook's turbulent flow friction approximations. Fluids 2(2), 15 (2017)

27. Eck, B.: Technische Strömungslehre. Springer-Verlag (1966)

28. Chen, N.H.: An explicit equation for friction factor in pipe. Ind. Eng. Chem. Fundam. 18(3), 296-297 (1979)

29. Round, G.F.: An explicit approximation for the friction factor-Reynolds number relation for rough and smooth pipes. Can. J. Chem. Eng. 58(1), 122-123 (1980)

30. Romeo, E., Royo, C., Monzón, A.: Improved explicit equations for estimation of the friction factor in rough and smooth pipes. Chem. Eng. J. 86(3), 369-374 (2002)

31. Buzzelli, D.: Calculating friction in one step. Mach. Des. 80(12), 54-55 (2008)

32. Gregory, J.M., McEnery, J.A.: Process-based friction factor for pipe flow. Open J. Fluid Dyn. 7(2), 219 (2017)

33. Brkić, D., Praks, P.: Accurate and efficient explicit approximations of the Colebrook flow friction equation based on the wright $\omega$-function. Mathematics $\mathbf{7}(1), 34$ (2019)

34. Brkić, D.: Review of explicit approximations to the Colebrook relation for flow friction. J. Petrol. Sci. Eng. 77(1), 34-48 (2011)

35. Genić, S., et al.: A review of explicit approximations of Colebrook's equation. FME Trans. 39(2), 67-71 (2011)

36. Giustolisi, O., Berardi, L., Walski, T.M.: Some explicit formulations of ColebrookWhite friction factor considering accuracy vs. computational speed. J. Hydroinform. 13(3), 401-418 (2011)

37. Winning, H.K., Coole, T.: Explicit friction factor accuracy and computational efficiency for turbulent flow in pipes. Flow Turbul. Combust. 90(1), 1-27 (2013)

38. Turgut, O.E., Asker, M., Coban, M.T.: A review of non iterative friction factor correlations for the calculation of pressure drop in pipes. Bitlis Eren Univ. J. Sci. Technol. 4(1), 1-8 (2014) 